\title{
Review with checklist of monocot in the herbarium of Iraq Natural History Museum
}

\author{
Khansaa Rasheed Al-Joboury* \\ Iraq Natural History Research Center and Museum, University of Baghdad, Baghdad, Iraq.
}

GSC Biological and Pharmaceutical Sciences, 2022, 18(02), 098-103

Publication history: Received on 29 December 2021; revised on 06 February 2022; accepted on 08 February 2022

Article DOI: https://doi.org/10.30574/gscbps.2022.18.2.0053

\begin{abstract}
This study was conducted to make an inventory of the monocot plants that were collected before and now which stored in the herbarium of Iraq Natural History Museum for identifying them. The herbarium contains avery large and varied number of plants from different parts in Iraq and for different and varied environments. The plants collected, arranged and identified using taxonomic keys specific to these families. Currently, the plant samples are in the herbarium of Iraq Natural History Museum to be an important scientific reference for all researchers inside and outside the country. With the identification of botanical scientists for each family, gender and year in which it was first diagnosed.
\end{abstract}

Keywords: Monocot; Herbarium; Iraq; Natural; History; Museum

\section{Introduction}

Monocotyledons (monocots) are the most important major radiations of angiosperm swhich recognized like a group since studies for seed structure with John Ray $(1682,1696,1703)$ at the seventeenth century[1,2]. One of the major differences between the monocots and the dicots is their possession for a single cotyledon (vs. usually only two in other angiosperms) Most monocots contain parallel leaf venation, floral parts with threes, sieve-tube plastids in several cuneate protein crystals, scattered vascular bundles for their stems, has no vascular-cambium-producing secondary phloem and secondary xylem $[3,4,5]$. In spite of their lack of a vascular cambium, some monocots, Also can become trees in increases the stem diameter by a novel process, The termed "anomalous" secondary growth, the plants are able to add more new vascular bundles and parenchyma to the primary bodyThat main division for Poales is that to the graminid and cyperid clades. Cyperaceae and Juncaceae are the important sister taxonomic, not intermingled like in some previous studies. Then their sister Thurniaceae follows. Poaceae is the most famous family in Iraq, Ecdeiocoleaceae Followed by the rest of the monocots families $[6,7]$.

\section{Material and methods}

All specimens of Monocot families in various habitats in Iraq which saved in the herbarium for Iraq Natural History Museum were written down. Used many taxonomic keys identification and diagnosed genera such as:[8]

\section{Results and discussion}

In this study checklist for monocot in the herbarium in Iraq Natural History Museum showed many families as fallow:

\footnotetext{
${ }^{*}$ Corresponding author: Khansaa Rasheed Al-Joboury

Iraq Natural History Research Center and Museum, University of Baghdad, Baghdad, Iraq. 


\subsection{Alismataceae 1799}

Alisma gramineum Lej. (1811)

Alisma lanceolatum With. (1796)

Alisma plantago-aquatica L. (1753)

Damasonium alisma Mill. (1768)

\subsection{Araceae 1789}

Biarum Schott 1832

\subsection{Asparagaceae 1789}

Hyacinthella nervosa (Bertol.) Chouard 1931

Leopoldia comosa (L.) Parl. 1847

Ornithogalum arcuatum Steven 1829

Ornithogalum magnum Krasch. \& Schischk. 1935

Ornithogalum montanum Cirillo 1811

Ornithogalum narbonense L. 1756

\subsection{Butomaceae 194}

Butomus umbellatus L. (1753)

\subsection{Cyperaceae 1789}

Cyperus laevigatus subsp. distachyos (All.) Ball (1878)

Blysmus Panz. ex Schult. (1824)

Bolboschoenus schmidii (Raymond) Holub (1973)

Bolboschoenus glaucus (Lam.) S.G.Sm. (1995)

Carex platyphylla J.Carey (1847)

Carex diluta M.Bieb. (1808)

Carex divisa Huds. (1762)

Cyperus glaber L. (1771)

Cyperus longus L. (1753)

Cyperus rotundus L. (1753)

Cyperus difformis L. (1756)

Cyperus fucosus K.L.Wilson (1991)

Cyperus glaber L. (1771)

Cyperus michelianus subsp. pygmaeus (Rottb.) Asch. \& Graebn. (1904)

Cyperus conglomeratus Rottb. (1773)

Fimbristylis dichotoma (L.) Vahl (1805)

Eleocharis palustris (L.) Roem. \& Schult. (1817)

Scirpoides holoschoenus (L.) Soják (1972)

Schoenoplectus lacustris (L.) Palla (1888)

\subsection{Gramineae (Poaceae) 1789}

Paspalum distichum L. (1759)

Aegilops columnaris Zhuk. (1928)

Aegilops crassa Boiss. 1846

Aegilops tauschii Coss. (1849)

Aegilops neglecta Req. ex Bertol. (1834)

Aeluropus lagopoides (L.) Thwaites (1864)

Aeluropus littoralis (Gouan) Parl. (1850)

Elymus repens (L.) Gould (1947)

Eremopyrum orientale (L.) Jaub. \& Spach (1851)

Agrostis gigantea Roth (1788)

Polypogon viridis (Gouan) Breistr. (1966)

Alopecurus apiatus Ovcz. (1934)

Alopecurus arundinaceus Poir. (1808)

Alopecurus myosuroides Huds. (1762) 
Alopecurus vaginatus (Willd.) Kunth (1833)

Gastridium ventricosum (Gouan) Schinz \& Thell. (1913)

Bromus rubens L. 1755

Stipagrostis plumosa Munro ex T.Anderson (1860)

Arrhenatherum elatius (L.) P.Beauv. ex J.Presl \& C.Presl. (1819)

Arrhenatherum kotschyi Boiss. (1846)

Arundo donax L. (1753)

Avena barbata Pott ex Link (1799)

Avena fatua L. (1753)

Avena ventricosa Balansa (1854)

Avena barbata Pott ex Link (1799)

Boissiera squarrosa (Sol.) Nevski (1934)

Briza humilis M.Bieb. (1808)

Bromus brachystachys Hornung (1833)

Bromus danthoniae Trin. 1831

Bromus japonicus Thunb. 1784

Bromus lanceolatus Roth 1797

Bromus modestus Renvoize 1994

Bromus scoparius L. 1755

Bromus tomentellus Boiss. 1846

Calamagrostis epigejos (L.) Roth 1788

Catabrosa P.Beauv., 1812

Catapodium rigidum (L.) C.E.Hubb. 1953

Chrysopogon gryllus (L.) Trin. 1820

Crypsis Aiton 1789

Ctenopsis De Not. 1847

Cymbopogon schoenanthus (L.) Spreng. 1815

Cynodon dactylon (L.) Pers. 1805

Cynosurus elegans Desf. 1798

Dactylis glomerata L. 1753

Dactyloctenium aegyptium (L.) Willd. 1809

Desmazeria Dumort. (1824)

Desmostachya bipinnata (L.) Stapf 1900

Digitaria sanguinalis (L.) Scop. 1771

Leptochloa fusca (L.) Kunth 1829

Echinochloa colona (L.) Link 1833

Echinochloa crus-galli (L.) P.Beauv. 1812

Elionurus hirtifolius Hack. 1889

Eragrostis basedowii Jedwabn. 1923

Eragrostis cilianensis (All.) Janch. 1907

Eragrostis diarrhena (Schult. \& Schult.f.) Steud. 1854

Eremopyrum bonaepartis (Spreng.) Nevski 1933

Eremopyrum distans (K.Koch) Nevski 1933

Eriochloa succincta (Trin.) Kunth 1833

Festuca arundinacea Schreb.

Heteranthelium piliferum (Sol.) Hochst. ex Jaub. \& Spach 1850

Hordeum marinum subsp. gussoneanum (Parl.) Thell. 1908

Hordeum marinum Huds. 1778

Imperata cylindrica (L.) Raeusch. 1797

Lolium persicum Boiss. \& Hohen. 1854

Lolium temulentum L. 1753

Milium pedicellare (Bornm.) Roshev. ex Melderis 1952

Piptatherum holciforme (M.Bieb.) Roem. \& Schult. 1817

Panicum miliaceum L. 1753

Parapholis incurva (L.) C.E.Hubb. 1946

Pennisetum divisum (Forssk. ex J.F.Gmel.) Henrard 1938

Phalaris minor Retz. 1783

Phalaris paradoxa L. 1763

Phleum alpinum L. 1753 
Phleum exaratum Griseb. 1846

Phleum pratense L. 1753

Phragmites australis (Cav.) Trin. ex Steud. 1841

Poa bulbosa L. 1753

Poa angustifolia L. 1753

Poa annua L. 1753

Poa trivialis L. 1753

Polypogon monspeliensis (L.) Desf. 1798

Saccharum strictum (Host) Spreng. 1815

Schismus barbatus (L.) Thell. 1907

Sclerochloa dura (L.) P.Beauv. 1812

Cutandia memphitica (Spreng.) Benth. 1881

Secale montanum Guss. 1825

Sorghum halepense (L.) Pers. 1805

Sphenopus divaricatus (Gouan) Rchb. 1830

Stipa barbata Desf. 1798

Stipa bromoides (L.) Dörfl. 1897

Stipa capensis Thunb. 1794

Brachypodium distachyon (L.) P.Beauv. 1812

Vulpia ciliata Dumort. 1824

\subsection{Hydrocharitaceae 1789}

Vallisneria spiralis L. 1753

\subsection{Iridaceae 1789}

Crocus cancellatus Herb. 1841

Gladiolus atroviolaceus Boiss. 1854

Gladiolus italicus Mill. 1768

Gladiolus italicus Mill. 1868

Iris persica L. 1753

Iris sisyrinchium L. 1753

\subsection{Ixioliriaceae 2015}

Ixiolirion tataricum (Pall.) Schult. \& Schult.f. (1829)

\subsection{Juncaceae 1789}

Juncus articulatus L. 1753

Juncus bufonius L. 1753

Juncus fontanesii J.Gay ex Laharpe 1825

Juncus inflexus L. 1753

\subsection{Liliaceae 1789}

Gagea bulbifera (Pall.) Salisb. 1806

\subsection{Orchidaceae 1789}

Epipactis helleborine (L.) Crantz 1769

Orchis mascula (L.) L. 1755

Neotinea tridentata (Scop.) R.M.Bateman, Pridgeon \& M.W.Chase 1997

\subsection{Potamogetonaceae}

Potamogeton crispus L. 1753

Stuckenia pectinata (L.) Börner 1912

Potamogeton perfoliatus L. 1753

Potamogeton pusillus L. 1753

Zannichellia palustris L. 1753 


\subsection{Ruppiaceae 2010}

Ruppia maritima L. 1753

\subsection{Typhaceae 1789}

Typha domingensis Pers. 1807

\subsection{Xanthorrhoeaceae 1829}

Asphodelus ramosus L. 1753

Asphodelus tenuifolius Cav. 1801

Monocots account about a quarter forangiosperm species richness and are among the very most economically and culturally important plants $[9,10]$, which have cereals (grasses), palms, orchids and lilies[11,12,13]. So it was observed that the increase in species richness in genera associated with attributes which bestow increased speciation rates and/or may be decreased extinction rates $[14,15]$

\section{Conclusion}

The study for our article showed that The presence of a large number of plants preserved in the herbarium of Iraq Natural History Museum, which date back to different periods of time, belonging to families of monocots The publication of these families is a way to facilitate the search for researchers of plant species belonging to these families, which are easy to obtain when published in a scientific journal.

\section{Compliance with ethical standards}

\section{Acknowledgments}

The author is grateful to the people who give me advice to complete this is an important achievement and provided me with some important books and resources to complete this research.

\section{Disclosure of conflict of interest}

No Conflict of interest.

\section{References}

[1] Hameeda B, Muhammad A, Asim M, Mustafa K, Inayat U R, Ali A. Morphological and Anatomical Studies on Some Monocot Xerophytes of District Karak, Pakistan. Middle-East Journal of Scientific Research. 2014; 22: 843-850.

[2] Parolin P. Submerged in darkness: adaptations to prolonged submergence by woody species of the Amazonian floodplains. Annals of Botany. 2009; 103: 359-376.

[3] Gostin IN. Anatomical and micromophological peculiarities of RanunculaceaePakistan Journal of Botany. 2011; 43: 811-820.

[4] Roghieh H, Fereshteh F, Mahboobeh A. Morphological and anatomical modifications in leaf, stem and roots of four plant species under boron deficiency conditions. Anales de Biología. 2012; 34: 15-29.

[5] Stahl Y, Simon R. Plant primary meristems: shared functions and regulatory mechanisms. Current Opinion in Plant Biology. 2010; 13: 53-58.

[6] Hajiboland R,Farhanghi F. Effect of low boron supply in turnip plants under drought stress. Biologia Plantarum. 2011; 55: 775-778.

[7] Kelly KS, Selena YS, Steven RM , Dashrath K, Deepak R, Amin G, Herminso V. Reinvestigating an enigmatic Late Cretaceous monocot: morphology, taxonomy, and biogeography of Viracarpon. Peer journal. 2018; 1-32.

[8] Townsend CC, Guest E, Al-Rawi. Flora of Iraq. Ministry of agriculture republic of Iraq. 1968; 9: 1-588.

[9] Stephens PR, Wiens JJ. Explaining species richness from continents to communities: the time-for-speciation effect in emydid turtles. The American Naturalist. 2003; 161: 112-128. 
[10] Trigas P, Panitsa M, Tsiftsis S. Elevational gradient of vascular plant species richness and endemism in Crete- the effect of post-isolation mountain uplift on a continental island system. PLoS ONE. 2013; 8: e59425.

[11] Schielzeth H. Simple means to improve the interpretability of regression coefficients. Methods in Ecology and Evolution. 2010; 1: 103-113.

[12] Schemske DW, Mittelbach GG, Cornell HV, Sobel JM, Roy K. Is there a latitudinal gradient in the importance of biotic interactions? Annual Review of Ecology, Evolution, and Systematics. 2009; 40: 245-269.

[13] Kuhn TS, Mooers AØ, Thomas GH. A simple polytomy resolver for dated phylogenies. Methods in Ecology and Evolution. 2011; 2: 427-436.

[14] Read J, Stokes A. Plant biomechanics in an ecological context. Amearican Journal of Botany. 2006; 93: 1546-1565.

[15] Grubb PJ, Jackson RV, Barberis IM, Bee JN, Coomes DA, Dominy NJ, et al. Monocot leaves are eaten less than dicot leaves in tropical lowland rain forests: correlations with toughness and leaf presentation. Annals of Botany. 2008; 101: 1379-1389. 